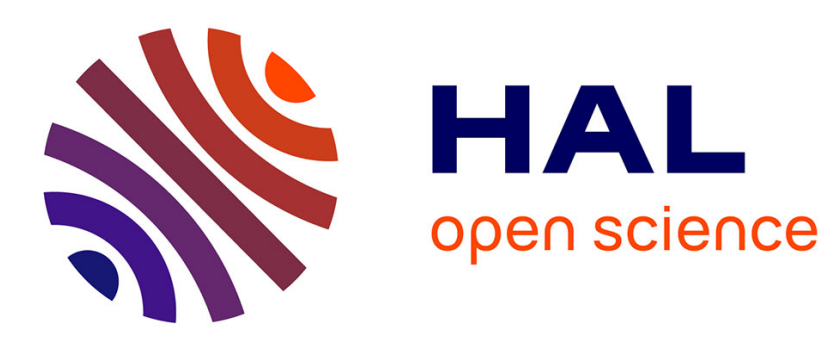

\title{
Influence des recommandations d'internautes: le role de la presence sociale et de l'expertise
}

Caroline Ardelet, Bérangère Brial

\section{To cite this version:}

Caroline Ardelet, Bérangère Brial. Influence des recommandations d'internautes: le role de la presence sociale et de l'expertise. Recherche et Applications en Marketing (French Edition), 2011, 26 (3), pp.4569. 10.1177/076737011102600303 . hal-01258971

\section{HAL Id: hal-01258971 \\ https://hal.science/hal-01258971}

Submitted on 28 Jan 2016

HAL is a multi-disciplinary open access archive for the deposit and dissemination of scientific research documents, whether they are published or not. The documents may come from teaching and research institutions in France or abroad, or from public or private research centers.
L'archive ouverte pluridisciplinaire HAL, est destinée au dépôt et à la diffusion de documents scientifiques de niveau recherche, publiés ou non, émanant des établissements d'enseignement et de recherche français ou étrangers, des laboratoires publics ou privés. 


\title{
Influence des recommandations d'internautes : le rôle de la présence sociale et de l'expertise
}

\author{
Caroline Ardelet \\ ATER \\ Université Paris-Dauphine \\ Dauphine Recherche en Management \\ DRM, UMR CNRS 7088 \\ Bérangère Brial \\ ATER \\ Université Paris-Dauphine \\ Dauphine Recherche en Management \\ DRM, UMR CNRS 7088
}

\section{RÉSUMÉ}

L'objectif est de comparer le pouvoir d'influence des recommandations d'internautes et des contenus institutionnels sur Internet. Nous montrons que les internautes sont plus influents que les sources institutionnelles lorsqu'ils semblent proches psychologiquement. Pour les recommandations en ligne, la présence sociale est un plus fort levier de persuasion que l'expertise.

Mots clés : Avis de consommateurs, blog, expertise, Internet, médias sociaux, présence sociale.

Les auteurs tiennent à remercier les professeurs Christian Pinson et Pierre Volle pour leurs remarques et suggestions, ainsi que les lecteurs anonymes qui, par leurs remarques constructives, ont permis une amélioration sensible de cet article.

Elles peuvent être contactées aux adresses électroniques suivantes :

Caroline_ardelet@yahoo.fr ; brial.berangere@neuf.fr 
Les médias sociaux jouent, à l'heure actuelle, un rôle fondamental dans le processus de décision d'achat d'un produit (Belvaux et Marteaux, 2007; Helme-Guizon, 2001 ; Larceneux, 2007 ; Kumar et Benbasat, 2006 ; Smith, Menon et Sivakumar, 2005). Depuis l'avènement du Web 2.0 et l'émergence d'une logique collaborative, les médias semblent avoir donné le pouvoir et la crédibilité aux masses (Pisani et Piotet, 2008 ; Surowiecki, 2005 ; Tapscott et Williams, 2007) ${ }^{1}$. Les contenus générés par les internautes sont présentés comme de meilleures sources d'information que les contenus institutionnels ${ }^{2}$. Même si les internautes contributeurs ne bénéficient d'aucune compétence particulière pour évaluer le produit, leurs « prises de parole » semblent plus pertinentes et plus crédibles que les informations émanant des sources institutionnelles (Bickart et Schindler, 2001).

Malgré l'essor de la logique contributive sur Internet, les recherches académiques sur l'influence des contenus générés par les utilisateurs par rapport aux contenus institutionnels restent pour le moment assez discordantes. D'une part, certaines recherches indiquent que l'expertise ${ }^{3}$ est le plus fort levier de la persuasion (Tractinsky et Rao, 2001). Les internautes accorderaient plus de crédit aux recommandations des experts et des marques car elles seraient plus compétentes pour évaluer le produit (Bounie et alii, 2008 ; Bronner et de Hoog, 2010; Eccleston et Griseri, 2008). D'autres part, des études suggèrent que l'impression d'une proximité psychologique avec les internautes, qui sont vus comme des pairs, rendrait leurs propos plus persuasifs (Brown et Reingen, 1987 ; Hass, 1981). Les recommandations d'internautes auraient alors plus d'influence sur les comportements des internautes qui les consultent que les recommandations des marques, des experts ou des journalistes (Bickart et Schindler, 2001).

Cette recherche contribue à expliquer ces contradictions dans la littérature et à montrer que, contrairement aux assertions actuelles des médias, les contenus

1. On va même jusqu'à parler d'empowerment des consommateurs (Firat, Dholakia et Venkatesh, 1995 ; Fuchs, Prandelli et Schreier, 2010), de consommacteur (Rebillard, 2007) ou de co-création des produits avec le consommateur (Prahalad et Ramaswamy, 2004).

2. «Les 10 mythes du Web 2 .0 », François Guillot, responsable Stratégies Internet du Groupe i\&e, Le Journal du Net du 23/01/2009.

3. Expert, du latin expertus, «qui a éprouvé», signifie «qui témoigne d'une compétence, habile, exercé» (Dictionnaire Larousse). générés par les internautes ne remplacent pas les contenus institutionnels. Pour cela, nous soutenons que le pouvoir d'influence des informations sur Internet dépend de l'impression de présence sociale que les internautes y perçoivent. En effet, la littérature montre que la confiance des internautes est renforcée lorsqu'ils ressentent de la présence sociale sur les sites internet (Kumar et Benbasat, 2002a, 2002b ; Gefen et Straub, 2004 ; Hassanein et Head, 2005, 2007). Une approche individuelle de la présence sociale, qui prend en compte la variabilité des réactions individuelles à l'égard des informations sur internet, doit permettre de nuancer la supériorité des contenus générés par les internautes par rapport aux contenus institutionnels.

Cet article propose de comparer l'attitude à l'égard d'un produit selon qu'il est recommandé par un internaute (contenu généré par un internaute) ou par le journaliste d'un blog spécialisé (contenu institutionnel), tout en soulignant le rôle de la présence sociale. Nous avons choisi de nous focaliser exclusivement sur la présence sociale véhiculée par des indices anthropomorphiques (incarnation du commentaire par une personne humaine via une photo, un nom et un langage parlé) (Gefen et Straub, 2003; Kumar et Benbasat, 2002a). Contrairement aux commentaires d'internautes qui sont de facto incarnés, les contenus institutionnels disponibles sur les blogs peuvent être des commentaires incarnés, lorsque le journaliste qui a rédigé le message est clairement identifié, ou des commentaires désincarnés, lorsqu'il reste anonyme. Par conséquent, cet article propose deux études. Dans l'étude 1, nous comparons l'avis d'un internaute, vecteur d'une présence sociale plus ou moins forte, à un article de type journalistique. Ce dernier étant désincarné, il est supposé sans présence sociale. Dans un deuxième temps, nous complétons les résultats obtenus en comparant l'avis d'internaute à l'avis d'un journaliste spécialisé. Ce dernier étant incarné, il est supposé véhiculer une présence sociale plus ou moins forte.

Nous avons choisi d'appliquer cette recherche au cas des blogs de parfums. Deux raisons ont motivé notre choix. Tout d'abord, les blogs étant considérés comme des média faibles en présence sociale (Kaplan et Haenlein, 2010), ils constituent un terrain d'étude neutre sur lequel il est possible de manipuler les vecteurs de présence sociale. De plus, le parfum est un produit pour lequel l'avis des tiers (spécialistes ou 
non) est déterminant dans la décision d'achat sans essai $^{4}$.

Nous définissons, dans une première partie, les fondements conceptuels qui nous permettent de poser les hypothèses de recherche. Nous présentons ensuite les deux études, puis les résultats obtenus. Enfin, l'article conclut sur les apports et limites de la recherche, et présente des voies de recherche futures.

\section{FONDEMENTS CONCEPTUELS ET HYPOTHÈSES}

\section{L'influence des recommandations d'experts et des non-experts}

Le web 2.0 a mis à la portée des internautes des outils leur permettant de donner leur avis sur les produits qu'ils consomment. Une nouvelle source d'information est ainsi venue compléter les sources institutionnelles que constituaient les informations fournies par les marques et les journalistes. Plusieurs recherches ont montré que les recommandations d'internautes avaient un effet positif sur les comportements de leurs lecteurs (Bickart et Schindler, 2001 ; Buhalis et Law, 2008 ; Chevalier et Mayzlin, 2006 ; Senecal et Nantel, 2004). Ainsi, elles facilitent l'achat (Smith, Menon et Sivakumar, 2005), suscitent un plus grand intérêt pour le produit (Bickart et Schindler, 2001 ; Wang et alii, 2007), et augmentent les intentions d'acheter (Ansari, Essegaier et Kohli, 2000 ; Hassanein et Head, 2005) et les ventes (Balagué et Florès, 2007 ; Clemons, Gao et Hitt, 2006 ; Duan et alii, 2008).

Si les auteurs s'accordent à dire que les recommandations d'internautes ont un effet positif sur les comportements des consommateurs qui les consultent, leur pouvoir de persuasion par rapport aux contenus institutionnels fait débat. Certains auteurs mettent en avant le manque d'expertise des inter-

4. Comme en témoigne la multiplication des recommandations d'internautes sur les forums dédiés (Osmoz.com, Beaute-test.com) et généralistes (Doctissimo.fr, Ciao.fr), ainsi que sur les blogs (Chroniques Olfactives, Esprit de Parfum), et les sites d'achats en ligne (Sephora.fr, Yves-Rocher.fr). nautes par rapport à une source institutionnelle. Ils reprennent la conclusion de Wilson et Sherrell (1993) selon laquelle « l'expertise ${ }^{5}$ est le plus fort levier de la persuasion » (p. 109), et l'appliquent aux relations médiatisées ${ }^{6}$ (Reeves et Nass, 1996). L'influence des recommandations d'internautes ne serait donc pas plus forte que celle des experts ou des marques (Bounie et alii, 2008; Bronner et de Hoog, 2010 ; Eccleston et Griseri, 2008). Mais pour d'autres, les internautes, qui sont vus comme des pairs, sont plus pertinents et plus crédibles que les informations institutionnelles fournies par les marques, les journalistes ou les experts (Bickart et Schindler, 2001). Cet article contribue à clarifier ces contradictions observées dans la littérature en identifiant dans quelles conditions le pouvoir de l'expertise prévaut par rapport à celui de la proximité.

\section{La présence sociale comme levier de la persuasion}

La première définition de la présence sociale remonte à Short, Williams et Christie en 1976. Elle caractérisait la capacité « subjective » d'un média ${ }^{7}$, à rendre saillant l'interlocuteur lors d'une discussion médiatisée par un écran (Short, Williams et Christie, 1976). Elle renvoyait donc à la capacité du média à restituer, aux yeux des interlocuteurs, les caractéristiques d'une communication interpersonnelle en face à face (Lombard et Ditton, 1997). À la fin des années 1990 , les chercheurs en systèmes d'information ont montré que la présence sociale pouvait être restituée à travers les médias sociaux, alors même que les interlocuteurs ne se voyaient pas. Ils ont alors identifié un ensemble de vecteurs de la présence sociale, au rang desquels figurent les vecteurs anthropomorphiques (Annexe A2).

La définition initiale de Short, Williams et Christie (1976) est particulièrement intéressante car elle souligne que la présence sociale est « subjective » (p. 66), c'est-à-dire que la tendance à ressentir de la présence dans un avis d'internaute varie selon les individus. Elle dépend notamment de ses caractéris-

5. Expert, du latin expertus «qui a éprouvé », signifie «qui témoigne d'une compétence, habile, exercé» (Dictionnaire Larousse).

6. Par média, Reeves et Nass entendent les ordinateurs, la télévision, l'internet.

7. À l'origine des appareils de visioconférence ou encore le vidéophone qui permettent de voir son interlocuteur à distance. 
tiques individuelles, comme, par exemple son genre (Gefen et Straub, 1997), son degré d'acceptation de la technologie (Davis, 1989 ; Komiak et Benbasat, 2006) ou encore ses motivations de shopping (Hassanein et Head, 2005). Une approche individuelle est donc indispensable.

Short, Williams et Christie (1976, p. 72) lient également la présence sociale à un autre concept issu de la psychologie sociale : celui de distance psychologique au sens de Wiener et Mehrabian (1968). L'étude des différentes définitions proposées depuis les années 70 (Annexe A1) montre que tous les auteurs ont, à la suite de Short, Williams et Christie (1976), repris sous des terminologies différentes cette notion de proximité psychologique, en parlant de « présence psychologique » (Lombard et Ditton, 1997) ou de «capacité du média à véhiculer l'expressivité et l'émotion » (Burke et Chindambaram, 1999, p. 566), ou encore de «compréhension, relation, implication, interaction » (Kumar et Benbasat, 2002a, p. 5). Ainsi, la présence sociale ressentie sur un blog donne aux internautes l'impression de converser avec d'autres internautes comme s'ils étaient à côté d'eux physiquement et en temps réel (Hassanein et Head, 2007 ; Kumar et Benbasat, 2002a). Ils ne ressentent pas la distance spatiale et temporelle qui les sépare. Si la présence sociale réduit la distance spatiale et temporelle entre les internautes, elle amoindrit également la distance sociale, c'est-à-dire les différences sociales (culture, passé...) . Ainsi, lorsque les internautes ressentent de la présence sociale, ils ont tendance à considérer que les autres internautes leur sont similaires ${ }^{8}$. « Même si le profil sociodémographique et les modes de vie de l'internaute contributeur ne sont pas similaires à ceux de l'internaute lecteur, [les internautes ont tendance à penser] que ce sont tous deux des consommateurs lambda » (Bickart et Schindler, 2001, p. 33).

Dans le monde physique, lorsqu'un produit est recommandé par une personne qui est jugée comme similaire, l'individu a tendance à penser que le produit correspond à ses attentes et à ce qu'il aime (Van Dolen, Dabholkar et de Ruyter, 2007). Le crédit accordé à une recommandation dépend ainsi de la similarité perçue entre la source du message et le récepteur (Hass, 1981 ; McGuire, 1969 ; Price, Feick

8. Le jargon Internet veut d'ailleurs que l'on nomme «pairs » les internautes, comme dans l'expression «peer to peer». Un pair est un homme de même condition, un égal. et Higie, 1989). En minimisant la distance temporelle, physique et sociale qui sépare le lecteur de l'émetteur, la présence sociale augmente le pouvoir d'influence de la recommandation. Ainsi, une recommandation de produit incite d'autant plus un internaute à aimer et à aller découvrir le produit en magasin qu'il se sent proche psychologiquement (spatialement, temporellement et socialement) de la personne qui recommande le produit.

\section{Hypothèses}

Dans le monde physique, les consommateurs semblent accorder plus de crédit aux messages émis par des personnes dont ils se sentent proches, surtout lorsqu'il s'agit de produits pour lesquels les préférences sont hétérogènes (Feick et Higie, 1992). En magasin, un conseiller perçu comme proche, même s'il est inexpérimenté, est plus influent qu'un conseiller distant expérimenté (Woodside et Davenport, 1974). La proximité avec la source est donc un plus fort levier de persuasion que l'expertise.

Dans la mesure où les caractéristiques des relations interpersonnelles en face à face peuvent être transférées aux relations médiatisées (Palmer, 1995 ; Reeves et Nass, 1996), nous faisons l'hypothèse que l'impression de proximité psychologique que les internautes peuvent ressentir avec l'émetteur d'une recommandation de produit conditionne davantage son impact sur les comportements que le degré d'expertise qui lui est accordé. Ainsi, un contenu institutionnel qui ne véhicule pas de présence sociale n'aurait pas plus d'influence sur les comportements des lecteurs qu'un contenu généré par un internaute. Au mieux, son pouvoir d'influence serait équivalent lorsque l'internaute lecteur se sent proche de la source institutionnelle. Nous supposons donc que l'appréciation d'un produit et l'intention d'aller le découvrir en magasin ne devraient pas varier significativement selon qu'il est recommandé par un internaute ou par une source institutionnelle dont les lecteurs se sentent proches. De plus, dès lors qu'il véhicule une forte présence sociale, un contenu généré par un internaute devrait avoir plus d'influence sur les internautes qu'un contenu institutionnel qui ne véhicule pas de présence sociale. S'il n'en véhicule pas, il n'a pas plus d'effet sur les comportements des internautes. Ceci nous conduit à formuler les hypothèses suivantes. 
Hla : Comparé à un contenu institutionnel à faible présence sociale, une recommandation d'internaute entraîne une meilleure appréciation du produit dès lors qu'elle véhicule une forte présence sociale.

$H 1 b$ : Comparé à un contenu institutionnel à faible présence sociale, une recommandation d'internaute entraîne une plus forte intention d'aller découvrir le produit en magasin dès lors qu'elle véhicule une forte présence sociale.

H2a: Une recommandation d'internaute à faible présence sociale entraîne la même appréciation $d u$ produit qu'un contenu institutionnel à faible présence sociale.

$H 2 b$ : Une recommandation d'internaute à faible présence sociale entraîne la même intention d'aller découvrir le produit en magasin qu'un contenu institutionnel à faible présence sociale.

H3a : Un contenu institutionnel à forte présence sociale entraîne la même appréciation du produit qu'une recommandation d'internaute, quel que soit son niveau de présence sociale.

$H 3 b$ : Un contenu institutionnel à forte présence sociale entraîne les même intentions d'aller découvrir le produit en magasin qu'une recommandation d'internaute, quel que soit son niveau de présence sociale.

\section{PREMIÈRE ÉTUDE : POUVOIR D'INFLUENCE DES RECOMMANDATIONS D'INTERNAUTES PAR RAPPORT AUX CONTENUS INSTITUTIONNELS DÉSINCARNÉS}

\section{Le design et la procédure}

L'objectif était de mesurer les attitudes des internautes à l'égard d'un produit selon qu'il était recommandé par un avis d'internaute ou par un article de type journalistique. À cette fin, nous avons retenu un design inter-sujet dans lequel les répondants étaient exposés, via une enquête en ligne, à une page de blog qui présentait un nouveau produit. Nous avons créé deux versions de cette page, et exposé les répondants à une des deux versions. Dans la première version, le produit était recommandé par un internaute. Dans la seconde version, le produit était décrit dans un article de type journalistique. Les observations obtenues suite à l'exposition à la recommandation d'internaute ont été divisées en deux groupes: d'une part les répondants ayant ressenti une forte présence sociale et d'autre part les répondants ayant ressenti une faible présence sociale. Aussi avons-nous demandé aux répondants, après exposition à la page du blog, d'évaluer sur l'échelle de Gefen et Straub (2004), que nous avons traduite en français ${ }^{9}$, la présence sociale ressentie. La variable obtenue après calcul d'un score factoriel suivait approximativement une loi normale. Après avoir éliminé, pour que les groupes soient bien contrastés, les observations contenues entre le $33^{\mathrm{e}}$ et le $66^{\mathrm{e}}$ percentile, nous avons retenu deux groupes : les répondants exposés à une recommandation perçue comme faible en présence sociale, et ceux exposés à une recommandation perçue comme forte en présence sociale.

\section{Création des stimuli}

Nous avons choisi de mettre en scène un blog et un parfum fictifs, inconnus et neutres. Nous avons donc créé deux versions d'une fiche produit présentant le parfum Eau parfumée sur le blog parfums.com (Annexes A4 et A5). Nous avons choisi pour ce parfum une senteur ${ }^{10}$ «pomme verte » et un flacon très classiques en parfumerie féminine ${ }^{11}$. Nous nous sommes assurées que les contenus des descriptions étaient identiques dans la recommandation de l'internaute et dans l'article. Les mêmes mots étaient utilisés (Annexe A3). Seules les structures de phrases et la ponctuation variaient afin d'être cohérentes avec la manière de s'exprimer de la source du message.

9. Avec l'aide de trois professeurs d'anglais (merci à Agnès Dussol, Zohra Ramouni et Arnaud Maria).

10. D'après un entretien avec Françoise Guermeur, responsable Évaluation olfactive chez Symrise, les senteurs fruitées sont très appréciées en France. Le vocabulaire lié aux odeurs de fruits est également plus familier et plus concret que le vocabulaire lié aux autres familles olfactives (comme par exemple les senteurs ambrées, aromatiques, boisées ou fleuries).

11. Préalablement à l'étude, nous avons présenté, en face à face, la photo de ce flacon de parfum à 20 étudiants en doctorat à l'Université. Nous leur avons demandé leur avis sur le flacon. La conclusion de ce pré-test qualitatif est que le flacon est neutre. Il ne suscite ni enthousiasme, ni rejet. 
Pour la page présentant la recommandation d'internaute, nous avons utilisé des vecteurs anthropomorphiques et éditoriaux de présence sociale (Annexe A2). Ainsi, l'internaute était incarné par une personne de sexe féminin, dénommée Marie. Ce prénom, très connu, suscite un fort agrément ${ }^{12}$. Elle était désignée par un pseudonyme typique du Web, Marie88, qui utilisait une combinaison prénom et numéro, et était représentée par une photo. Un prétest auprès de 20 étudiants en doctorat à l'Université nous avait indiqué que, sur la base de cette photo, Marie était perçue comme une jeune femme ayant la trentaine, plutôt jolie et sympathique. De plus, la recommandation était rédigée dans un langage parlé (avec des phrases déstructurées et de nombreuses marques de ponctuation) comme il est d'usage dans les avis d'internautes.

La photo du produit était placée sur la gauche et le texte (soit la recommandation de Marie88 soit l'article de type journalistique) sur la droite. Afin de décider de l'emplacement de la photo par rapport au texte, nous nous sommes appuyés sur les recherches sur la spécialisation des hémisphères cérébraux. Ces recherches indiquent qu'il est préférable de placer l'image à gauche du texte plutôt qu'à droite (Ellis et Miller, 1981; Janiszewski, 1988). En effet, le texte, lorsqu'il est situé dans le champ visuel droit, est plus aisément traité par l'hémisphère gauche. À l'inverse, l'image, lorsqu'elle est située dans le champ visuel gauche, est plus aisément traitée par l'hémisphère droit, zone spécialisée dans le traitement des informations visuelles. Ceci corrobore par ailleurs les pratiques managériales sur les forums et les blogs.

Pour la page présentant l'article de type journalistique, nous avons utilisé un langage soutenu (avec des phrases composées d'un sujet, d'un verbe et éventuellement d'un complément). Aucune précision n'était donnée sur la personne qui avait rédigé l'article.

\section{Les participants}

Seules des femmes ont été interrogées, car la littérature les dit plus sensibles à la présence sociale que

12. Sur le site prenoms.com, le prénom Marie est évalué par 1166 internautes et obtient la note d'appréciation de 4,13 sur 5 . les hommes (Gefen et Straub, 1997), et $76 \%$ des achats de parfums sont effectués par des femmes ${ }^{13}$. Grâce à un effet boule de neige à partir des bases de données dont disposaient les chercheurs, 174 répondants ont été contactés par mail pour répondre à une l'enquête en ligne, 70 d'entre eux ont été exposés à la contribution de membres et 104 d'entre eux à l'article de type journalistique. La procédure d'élimination des individus ayant ressenti une présence sociale moyenne (c'est-à-dire ceux pour lesquels les réponses étaient comprises entre le $33^{\mathrm{e}}$ et le $66^{\mathrm{e}}$ percentile) nous a conduit à éliminer 50 réponses. $\mathrm{Au}$ final, cette procédure a permis de récolter 124 observations (75 observations pour l'article, 22 pour la recommandation à faible présence sociale et 27 pour la recommandation à forte présence sociale).

\section{Échelles de mesure utilisées}

\section{La présence sociale}

Nous avons utilisé l'échelle monodimensionnelle de présence sociale de Gefen et Straub (2004), que nous avons traduite en français (Annexe A6). Nous avons ensuite calculé un score factoriel reflétant l'impression de présence sociale que chaque internaute perçoit sur la page Internet à laquelle il est exposé.

\section{L'attitude à l'égard du produit}

D'une part, nous avons mesuré les réactions émotionnelles en demandant directement aux répondants s' «ils aiment le parfum qui est présenté sur la page ». Ils étaient invités à se prononcer sur une échelle en 7 points. D'autre part, nous avons mesuré les prédispositions à agir à l'égard du parfum via trois items (cette fiche descriptive vous donne-t-elle envie de sentir ce nouveau parfum ? / Si vous voyiez ce parfum dans un magasin, iriez-vous le sentir? / Si vous aviez besoin d'acheter un parfum, essaieriez-vous de trouver ce parfum dans le magasin pour le sentir?).

13. Le marché des parfums, octobre 2007, étude réalisée pour le compte de Mondadori France Publicité, http://www.mondadoripub.fr/ 
Les répondants étaient invités à répondre sur des échelles en 7 points. À partir de ces trois items, nous avons formé un score reflétant les comportements d'approche à l'égard du parfum.

\section{Les variables de contrôle}

Tout d'abord, nous avons mesuré le degré d'expertise accordée à la source du message à travers une échelle mono-item qui consistait à demander aux internautes d'indiquer, sur une échelle de Likert en 7 points, à quel point ils pensaient que l'avis émanait d'une personne experte en parfums.

Ensuite, la littérature montre que la familiarité des mots facilite leur traitement perceptivo-cognitif. Plus un mot survient fréquemment dans le langage, plus les temps de réaction qui lui sont associés sont courts dans des épreuves de lecture à voix haute, de décision lexicale et de catégorisation lexicale ou sémantique (Allen, McNeal et Kvak, 1992 ; Desrochers, Paivio et Desrochers, 1989; Monsell, Doyle et Haggard, 1989 ; Paap et Johansen, 1994). Dans la mesure où la recommandation d'internaute est rédigée dans un langage parlé et que l'article est rédigé dans un style plus soutenu, nous avons veillé à ce qu'il n'y ait pas de différence significative dans la facilité de compréhension des messages. À cette fin, les répondantes devaient se prononcer sur une échelle sémantique différentielle en 7 points. Par ailleurs, nous nous sommes également assurées que l'effort cognitif nécessaire pour traiter les messages était identique. Nous avons donc utilisé l'échelle ressource demand de Keller et Block (1997) ${ }^{14}$.

14. Les cinq items de l'échelle ont été administrés sous la forme d'échelle de Likert en 7 points. Les répondants devaient se prononcer sur la difficulté de compréhension, la nécessité d'être concentré, le fait que le message soit facile à comprendre et à suivre et qu'il attire l'attention.

\section{Résultats}

\section{Les variables de contrôle}

Les résultats montrent que Marie88 est jugée moins experte que le journaliste ayant rédigé l'article de type journalistique $(\mathrm{p}<0,05)$ (Tableau 1). Par ailleurs, la recommandation de Marie88 est aussi facile à comprendre que l'article de type journalistique. Les différences de moyennes ne sont pas significatives $(\mathrm{p}=0,357)$ (Tableau 1). Les répondants estiment que le traitement de la recommandation de Marie88 demande autant de ressources cognitives que celui de l'article de type journalistique. Les différences de moyennes ne sont pas significatives ( $\mathrm{p}=$ 0,568) (Tableau 1).

\section{Le pouvoir d'influence des recommandations d'internautes par rapport à celui des articles de type journalistique}

Les deux premières hypothèses supposaient que, comparée à un article de type journalistique ne véhiculant pas de présence sociale, une recommandation d'internaute entraînait une meilleure appréciation (H1a) et une meilleure intention d'aller découvrir le produit (H1b) dès lors qu'elle véhiculait une forte présence sociale. Nous avons également supposé que l'appréciation du produit (H2a) et l'intention d'aller le découvrir en magasin (H2b) 2HhHne variaient pas significativement selon qu'il était présenté dans une recommandation d'internaute à faible présence sociale ou dans un article de type journalistique.

Des tests de différences de moyennes indiquent qu'il n'y a pas de différence significative dans l'appréciation du produit et dans l'intention d'aller le découvrir selon qu'il est recommandé par l'article de

Tableau 1. - Variables de contrôle pour l'étude 1

\begin{tabular}{|c|c|c|c|c|c|c|}
\hline \multirow{2}{*}{ Source } & \multirow{2}{*}{$\begin{array}{c}\text { Différence de moyenne } \\
\text { (article vs internaute) }\end{array}$} & \multicolumn{2}{|c|}{ Test de Levene } & \multicolumn{3}{|c|}{ Test t } \\
\cline { 3 - 6 } & 0,334 & 0,466 & 0,496 & $-0,924$ & 123 & 0,357 \\
\hline Article journalistique & $-0,108$ & 0,277 & 0,600 & 0,572 & 122 & 0,568 \\
\hline Contribution de Marie88 & $-0,902$ & 4,573 & 0,034 & 3,125 & 122 & 0,002 \\
\hline Article journalistique & Contribution de Marie88 & & & & Sig. & 122 \\
\hline Article journalistique & & & & & &
\end{tabular}




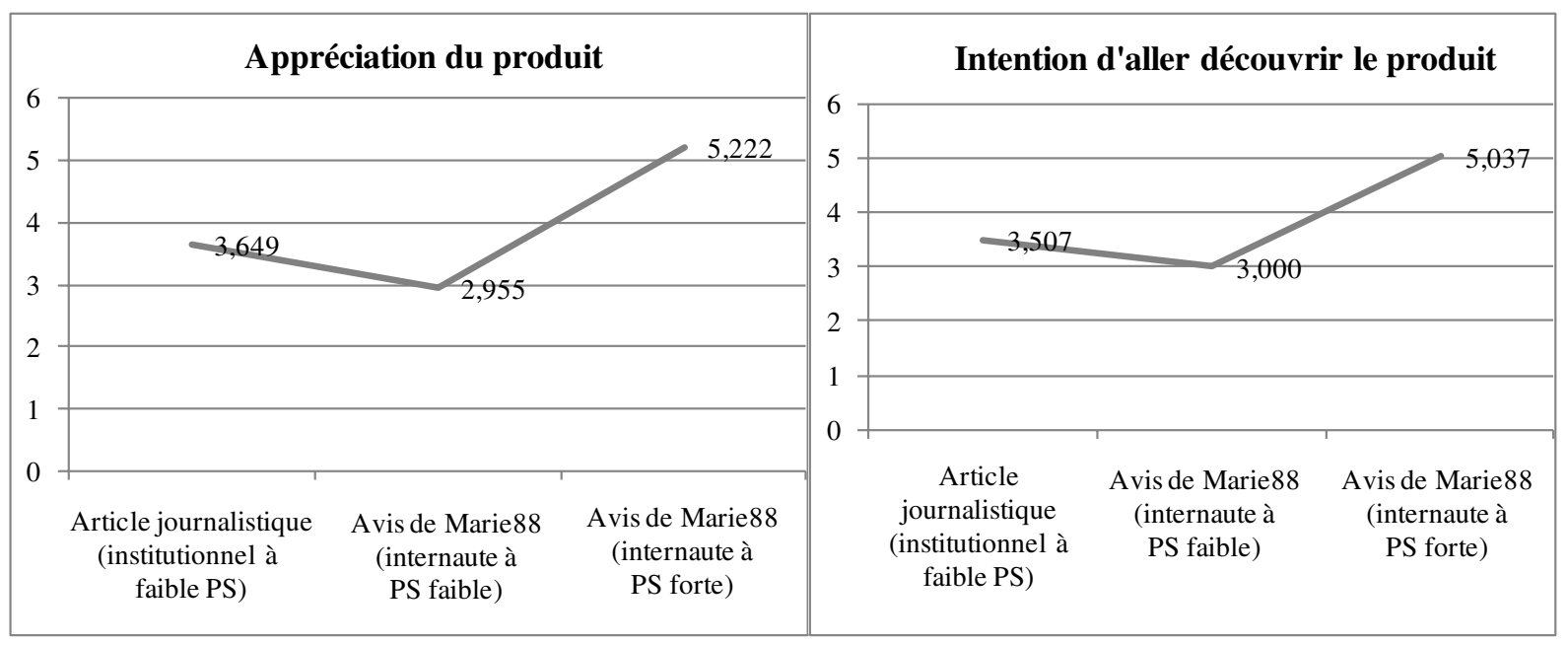

Figure 1

type journalistique ou par Marie88 ( $\mathrm{p}>0,05)$ (Tableau 2). Ainsi, l'expertise de la source n'a pas d'effet direct. En revanche, lorsque le produit est recommandé par Marie88 et que l'internaute perçoit une forte présence sociale, l'appréciation du produit et l'intention d'aller découvrir le produit sont plus fortes que lorsque le produit est décrit par un article de type journalistique (Figure 1). La recommandation de Marie88 augmente l'appréciation du produit et les intentions d'aller le découvrir en magasin lorsque son avis dégage de la présence sociale $(\mathrm{p}<0,05)$ (Tableau 3). Les hypothèses H1a et H1b sont validées. De plus, lorsque le produit est recommandé par Marie88 et que l'internaute perçoit une faible présence sociale, l'appréciation du produit et l'intention d'aller le découvrir en magasin ne sont pas significativement différentes de celles obtenues lorsque le produit est décrit dans un article de type journalistique $(p>0,05)$ (Tableau 3). Les hypothèses $\mathrm{H} 2 \mathrm{a}$ et $\mathrm{H} 2 \mathrm{~b}$ sont validées.

\section{Discussion des résultats de la première étude}

La mise en avant du rôle de la présence sociale permet de réconcilier les précédentes recherches apparemment discordantes. La supériorité des informations d'internautes par rapport aux informations données par les sources institutionnelles n'est pas systématique. Dès lors que les internautes contributeurs paraissent proches psychologiquement, grâce à une forte impression de présence sociale ressentie dans leur commentaire, les lecteurs jugent que les recommandations d'internautes sont plus pertinentes et plus crédibles que celles des articles de type journalistique. En revanche, si les internautes contributeurs paraissent distants, alors les recommandations d'internautes n'ont pas plus d'impact que celles des articles de type journalistique. 

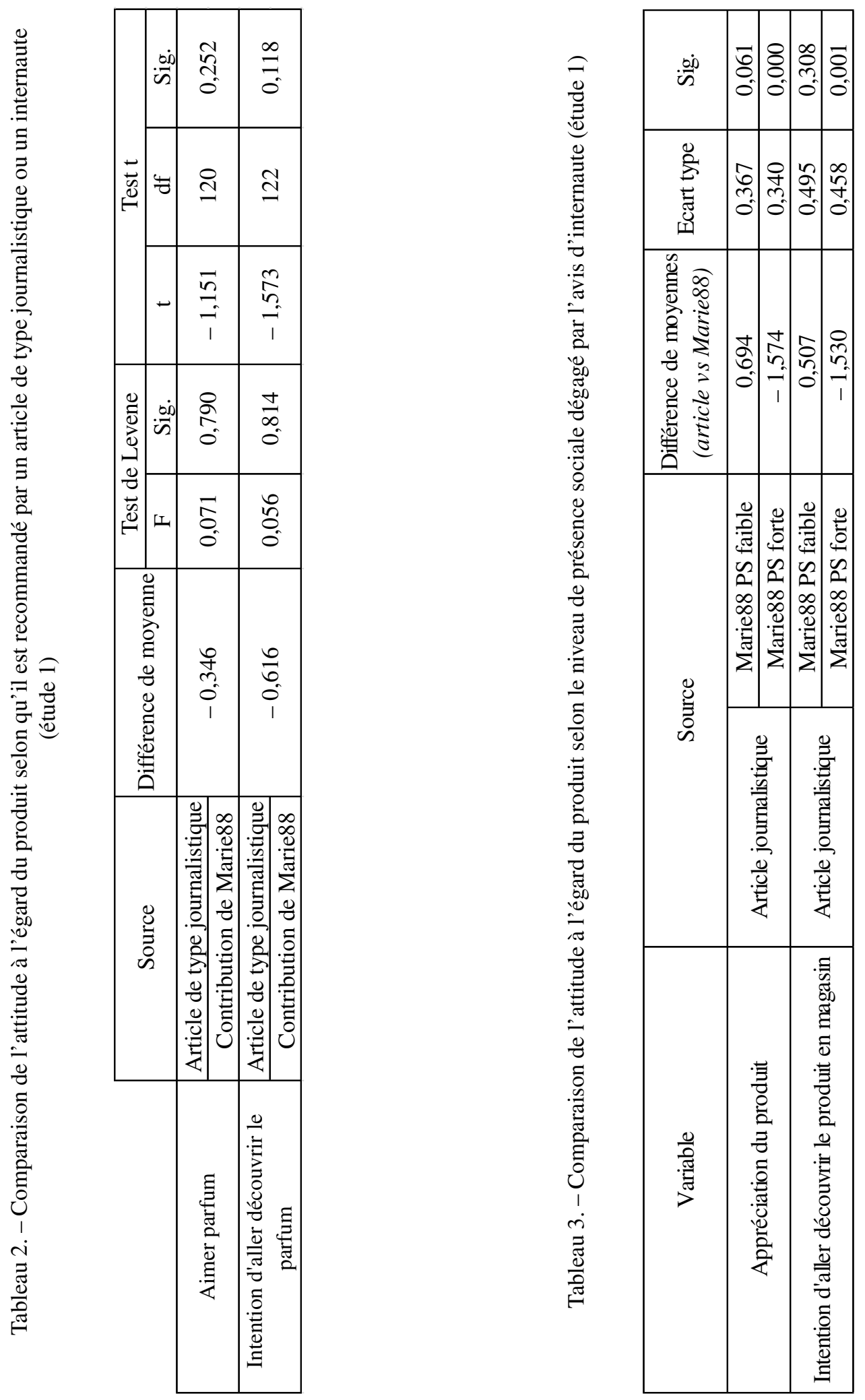


\section{DEUXIÈME ÉTUDE : POUVOIR D'INFLUENCE DES RECOMMANDATIONS D'INTERNAUTES PAR RAPPORT AUX CONTENUS INSTITUTIONNELS INCARNÉS}

\section{Le design, la procédure et les participants}

Cette deuxième étude visait à compléter les résultats de la première étude en s'intéressant aux contenus institutionnels incarnés par une personne humaine susceptibles de véhiculer de la présence sociale. Nous avons choisi de reprendre le blog parfums.com, ainsi que le nom et le flacon du parfum Eau Parfumée et de comparer la recommandation d'un internaute à celle d'un journaliste employé par le blog. Comme dans la première étude, les répondantes étaient exposées en ligne, via le logiciel wyzuforms, à une des deux versions de la fiche produit que nous avions créées. Dans la première version, le parfum était recommandé par l'avis d'internaute. Dans la seconde version, le produit était recommandé par l'avis de spécialiste. Les contenus des deux avis étaient identiques.

Cette deuxième étude a été administrée auprès des répondantes qui avaient accepté, lors de l'étude 1 , de participer à une deuxième étude sur les parfums. Les répondantes qui avaient été exposées au contenu institutionnel désincarné (article de type journalistique) ont été exposées à l'avis d'internaute et celles exposées à l'avis d'internaute (recommandation de Marie88) ont été exposées au contenu institutionnel incarné (avis de spécialiste). Toutes les répondantes d'une même condition ont donc été exposées au même traitement lors de l'étude 1. Nous étions conscients que cette première étude constituait une tâche d'amorçage qui pouvait influencer les résultats de l'étude 2 . Cependant, dans la mesure où toutes les répondantes d'une même condition étaient sujettes à la même amorce, nous avons supposé que les biais étaient limités.

Cette procédure nous a permis de contacter 69 répondantes, 30 d'entre elles étant exposées à l'avis d'internaute et 39 d'entre elles étant exposées à l'avis de spécialiste. La base de données obtenue a été épurée de 5 répondantes dont les réponses présentaient des incohérences. Quatre d'entre elles avaient attribué la même note à plus de $50 \%$ des questions (note de 7 pour deux des répondantes, notes de 1 et 2 pour les deux autres répondantes). Ces répondantes semblaient avoir attribué des notes de manière systématique, sans prendre le temps de nuancer leur jugement. La dernière répondante supprimée avait eu des problèmes de compréhension de l'échelle de présence sociale qui présentait de nombreuses réponses manquantes. Nous avons ensuite adopté la même procédure que dans la première étude pour diviser les observations en deux groupes : d'une part les répondants ayant ressenti une forte présence sociale et d'autre part les répondants ayant ressenti une faible présence sociale. Le nombre d'observations récoltées étant plus petit que dans la première étude, nous avons éliminé les réponses des répondants dont le score factoriel était compris entre le $40^{\mathrm{e}}$ et le $60^{\mathrm{e}}$ percentile. Nous avons ainsi éliminé 13 réponses. Nous avons ainsi obtenu un plan factoriel à quatre conditions : 2 (avis d'internaute/avis de spécialiste) $\times 2$ (faible présence sociale ressentie/forte présence sociale ressentie). Au final, nous avons retenu 51 réponses, 20 exposés à l'avis d'internaute et 31 à l'avis de spécialiste.

\section{Création des stimuli}

Nous avons utilisé la même page de blog parfums.com et le même flacon d'Eau parfumée (Annexes A4 et A5). Nous avons légèrement modifié la senteur du parfum, en mettant en avant une senteur « cocktail de fruits ». Pour créer les deux versions de la fiche produit, nous avons repris le même graphisme que les fiches produits de la première étude, avec la photo du produit positionnée à gauche du texte descriptif. Comme dans la première étude, nous avons utilisé des vecteurs anthropomorphiques et éditoriaux de présence sociale (Annexe A2). Ainsi, le spécialiste et l'internaute étaient incarnés par des personnes de sexe féminin, dénommées Alice ${ }^{15}$. Elles s'exprimaient toutes deux dans un langage parlé, et étaient représentées par une même photo qui, d'après un pré-test auprès de 20 étudiants en doctorat à l'Université, les présentaient comme des jeunes femmes ayant la trentaine, plutôt jolies et sympathiques.

15. Ce prénom, très connu, suscite un fort agrément. Sur le site prenoms.com, le prénom Alice est évalué par 750 internautes et reçoit une note de 4,34/5. 
Afin de clairement distinguer l'internaute du spécialiste, nous avons manipulé la manière de présenter l'identité du contributeur et le niveau de langage employé dans la recommandation. Ainsi, l'internaute lambda était présentée par un pseudonyme, Alice55, et s'exprimait dans un langage parlé familier, avec un verbe à l'impératif interpellant le lecteur et de nombreux points d'exclamation (Annexe A3). La spécialiste était présentée quant à elle par son nom et son prénom, Alice Audiart, et s'exprimait dans un langage parlé plus soutenu. Sa fonction, « rédactrice en chef de la rubrique Parfums », était précisée à côté de son nom. La désignation par le prénom et le nom de famille, et l'affichage d'une fonction d'experte dans les parfums appuyaient l'expertise de cette personne.

\section{Échelles de mesure utilisées}

Nous avons utilisé les mêmes échelles que dans la première étude, à savoir l'échelle de présence sociale Gefen et Straub (2004) traduite en français (Annexe A6), la fréquence subjective des mots utilisés, la quantité de ressources nécessaires pour traiter le message, l'expertise perçue de la source du message, l'appréciation du parfum ainsi que le score de comportement d'approche créé pour la première étude.

\section{Résultats}

\section{Les variables de contrôle}

Les résultats montrent qu'Alice Audiart est jugée plus experte qu'Alice55 ( $\mathrm{p}<0,05$ ) (Tableau 4). De plus, l'avis d'Alice Audiart est aussi facile à com- prendre que celui d'Alice55. Les différences de moyennes ne sont pas significatives $(\mathrm{p}=0,551)$ (Tableau 4). Les répondants estiment que le traitement de l'avis d'Alice Audiart demande autant de ressources cognitives que celui d'Alice55. Les différences de moyennes ne sont pas significatives $(\mathrm{p}=0,745)$ (Tableau 4).

\section{Le pouvoir d'influence des recommandations d'internautes par rapport aux recommandations des spécialistes}

Cette deuxième étude nous permet de répliquer les résultats obtenus à l'étude 1 . Comme dans l'étude 1 , l'expertise de la source du message n'a pas d'effet direct sur l'appréciation et l'intention d'aller découvrir le produit en magasin. Il n'y a pas de différence significative dans l'attitude à l'égard du produit selon qu'il est recommandé par un internaute lambda (Alice55) ou par un spécialiste (Alice Audiart) ( $p>0,05)$ (Tableau 5). L'effet de l'expertise dépend du niveau de présence sociale que les contenus dégagent. L'interaction existant entre la présence sociale ressentie et la source du message est significative (en unilatéral) pour l'appréciation du produit $(\mathrm{p}<0,1)$ et pour l'intention d'aller découvrir le produit $(\mathrm{p}<0,1)$ (Tableau 7). Ainsi, la recommandation d'internaute est plus influente que celle du spécialiste lorsque la recommandation d'internaute a une forte présence sociale et celle du spécialiste une faible présence sociale. Dans les autres conditions de présence sociale, la recommandation d'internaute et celle du contenu institutionnel ont un impact équivalent sur les internautes (Figure 2).

Des tests de différences de moyennes permettent de valider, comme dans l'étude 1, les hypothèses

Tableau 4. - Variables de contrôle pour l'étude 2

\begin{tabular}{|c|c|c|c|c|c|c|}
\hline \multirow{2}{*}{ Source } & \multirow{2}{*}{$\begin{array}{c}\text { Différence de moyenne } \\
\text { (Alice55 vs Alice Audiart) }\end{array}$} & \multicolumn{2}{|c|}{ Test de Levene } & \multicolumn{3}{|c|}{ Test $\mathrm{t}$} \\
\hline & & $\mathrm{F}$ & Sig. & $\mathrm{t}$ & df & Sig. \\
\hline Contribution d'Alice55 & \multirow{2}{*}{1,123} & \multirow{2}{*}{1,558} & \multirow{2}{*}{0,218} & \multirow{2}{*}{$-0,601$} & \multirow{2}{*}{49} & \multirow{2}{*}{0,551} \\
\hline Contribution d'Alice Audiart & & & & & & \\
\hline Contribution d'Alice55 & \multirow{2}{*}{$-0,090$} & \multirow{2}{*}{0,315} & \multirow{2}{*}{0,578} & \multirow{2}{*}{$-0,328$} & \multirow{2}{*}{46} & \multirow{2}{*}{0,745} \\
\hline Contribution d'Alice Audiart & & & & & & \\
\hline Contribution d'Alice55 & \multirow{2}{*}{$-0,902$} & \multirow{2}{*}{0,285} & \multirow{2}{*}{0,596} & \multirow{2}{*}{$-2,364$} & \multirow{2}{*}{49} & \multirow{2}{*}{0,022} \\
\hline Contribution d'Alice Audiart & & & & & & \\
\hline
\end{tabular}


H1a, H1b, H2a et H2b. Ainsi, lorsque le produit est présenté par Alice55 à travers une recommandation qui véhicule une forte présence sociale, il est plus apprécié et les intentions d'aller le découvrir en magasin plus nombreuses que lorsqu'il est présenté par Alice Audiart à travers une recommandation qui véhicule une faible présence sociale $(\mathrm{p}<0,05$ pour l'appréciation du produit et $\mathrm{p}<0,1$ pour l'intention d'aller le découvrir en magasin) (Tableau 6). H1a et $\mathrm{H} 1 \mathrm{~b}$ sont validées. De plus, lorsque le produit est recommandé par Alice55 et que l'internaute perçoit une faible présence sociale, l'appréciation du produit et l'intention d'aller le découvrir en magasin ne sont pas significativement différentes de celles obtenues lorsque le produit est recommandé par Alice Audiart et que l'internaute perçoit une faible présence sociale ( $p>0,05$ ) (Tableau 6). H2a et H2b sont validées.
Cette deuxième étude visait également à tester les hypothèses $\mathrm{H} 3 \mathrm{a}$ et $\mathrm{H} 3 \mathrm{~b}$ qui supposaient qu'un contenu institutionnel à forte présence sociale entrấnait la même appréciation du produit $(\mathrm{H} 3 \mathrm{a})$ et la même intention d'aller le découvrir $(\mathrm{H} 3 \mathrm{~b})$ qu'une recommandation d'internaute, quel que soit son niveau de présence sociale. Un test de différences de moyennes indique que le spécialiste (Alice Audiart) a autant d'influence que l'internaute lambda (Alice55) lorsque sa recommandation dégage une forte présence sociale. Dans ce cas, la recommandation d'Alice Audiart déclenche la même appréciation et la même intention d'aller découvrir le produit qu'Alice 55 (Tableau 6). H3 et $\mathrm{H} 4$ sont validées.

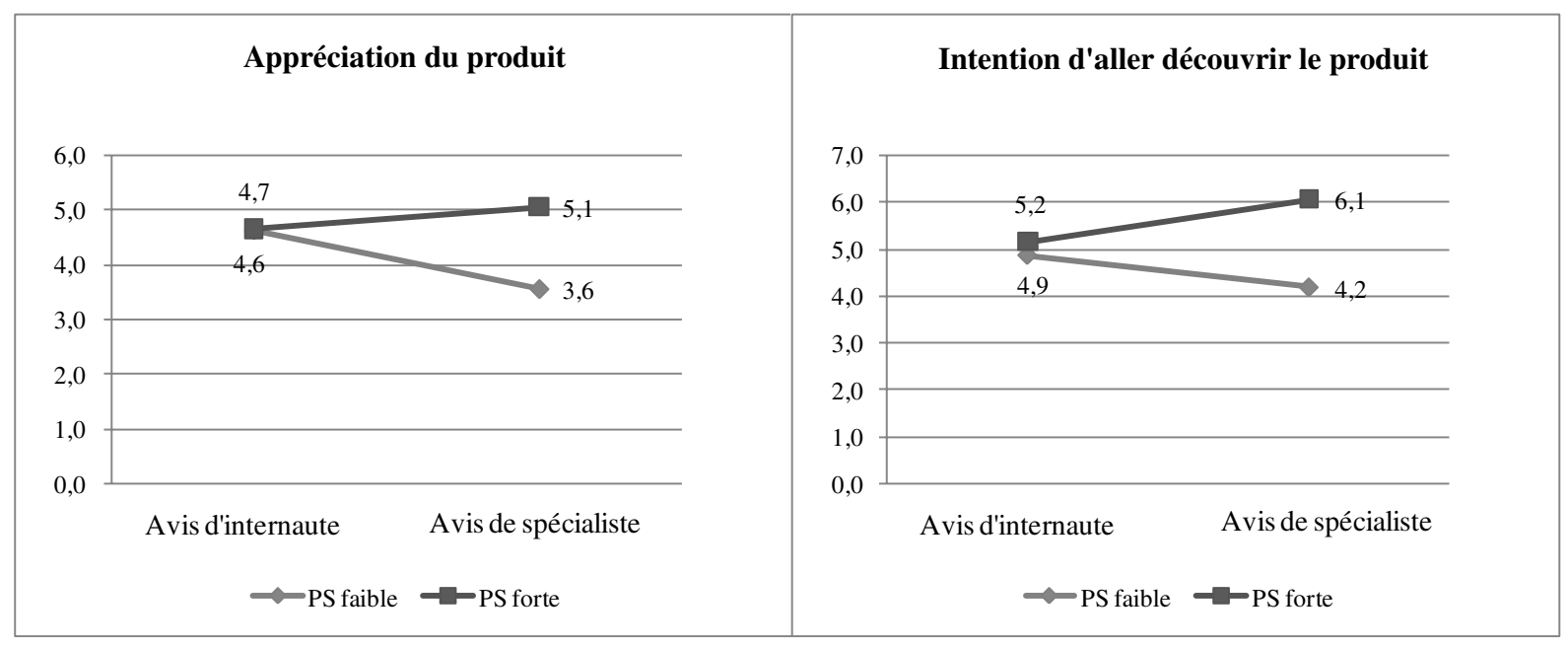

Figure 2

Tableau 5. - Comparaison de l'attitude à l'égard du produit selon qu'il est recommandé par un internaute ou un spécialiste (étude2)

\begin{tabular}{|c|c|c|c|c|c|c|c|}
\hline & \multirow{2}{*}{ Source } & \multirow{2}{*}{ Différence de moyenne } & \multicolumn{2}{|c|}{ Test de Levene } & \multicolumn{3}{|c|}{ Test $\mathrm{t}$} \\
\hline & & & $\mathrm{F}$ & Sig. & $\mathrm{t}$ & $\mathrm{df}$ & Sig. \\
\hline \multirow{2}{*}{ Aimer parfum } & Contribution d'Alice55 & \multirow{2}{*}{0,360} & \multirow{2}{*}{0,002} & \multirow{2}{*}{0,962} & \multirow{2}{*}{0,833} & \multirow{2}{*}{49} & \multirow{2}{*}{0,409} \\
\hline & Contribution d'Alice Audiart & & & & & & \\
\hline \multirow{2}{*}{$\begin{array}{c}\text { Intention d'aller découvrir le } \\
\text { parfum }\end{array}$} & Contribution d'Alice55 & \multirow{2}{*}{$-0,255$} & \multirow{2}{*}{0,249} & \multirow{2}{*}{0,620} & \multirow{2}{*}{$-0,538$} & \multirow{2}{*}{46} & \multirow{2}{*}{0,593} \\
\hline & Contribution d'Alice Audiart & & & & & & \\
\hline
\end{tabular}




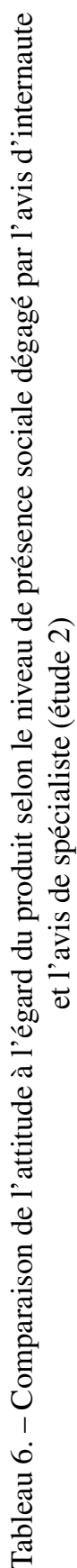

\begin{tabular}{|c|c|c|c|c|c|c|c|c|c|c|c|c|c|c|c|c|c|c|}
\hline id & 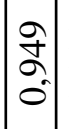 & $\begin{array}{lll}\infty & 0 \\
0 \\
0 \\
0\end{array}$ & 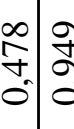 & & $\left|\begin{array}{c}0 \\
6 \\
0 \\
0\end{array}\right|$ & $\begin{array}{lll}\infty & 0 \\
0 \\
0 \\
0\end{array}$ & $\begin{array}{l}0 \\
0 \\
b\end{array}$ & $\left|\begin{array}{c}\infty \\
0 \\
0 \\
0\end{array}\right|$ & & $\begin{array}{l}\infty \\
\vdots \\
0 \\
0 \\
0\end{array}$ & & $\begin{array}{l}\infty \\
0 \\
0 \\
0 \\
0 \\
0\end{array}$ & & $\begin{array}{l}2 \\
0 \\
0\end{array}$ & & & & 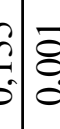 \\
\hline 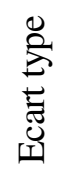 & fo & $\begin{array}{l}0 \\
0 \\
0 \\
0\end{array}$ & 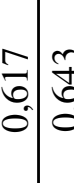 & {$\left[\begin{array}{l}\infty \\
n \\
n \\
n\end{array}\right.$} & 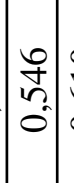 & $\begin{array}{l}\frac{0}{0} \\
\stackrel{0}{0}\end{array}$ & $\begin{array}{c}0 \\
0 \\
c \\
0 \\
0\end{array}$ & $\begin{array}{l}7 \\
6 \\
0\end{array}$ & 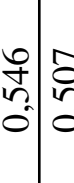 & {$\left[\begin{array}{l}\infty \\
0 \\
0 \\
0 \\
0\end{array}\right.$} & 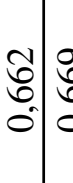 & $\begin{array}{l}0 \\
\vdots \\
\vdots \\
0 \\
0 \\
0\end{array}$ & $\begin{array}{l} \pm \\
0 \\
\text { in }\end{array}$ & $\begin{array}{cc}1 \\
\vdots \\
\vdots \\
\vdots\end{array}$ & 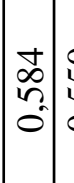 & & & \\
\hline
\end{tabular}

离

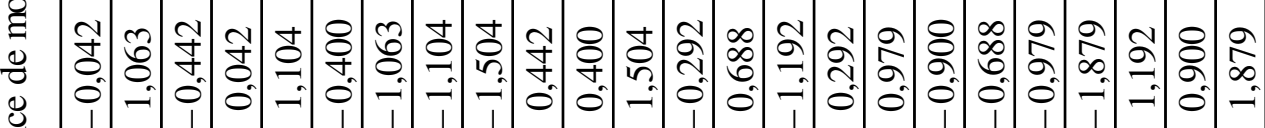

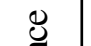

这

ส

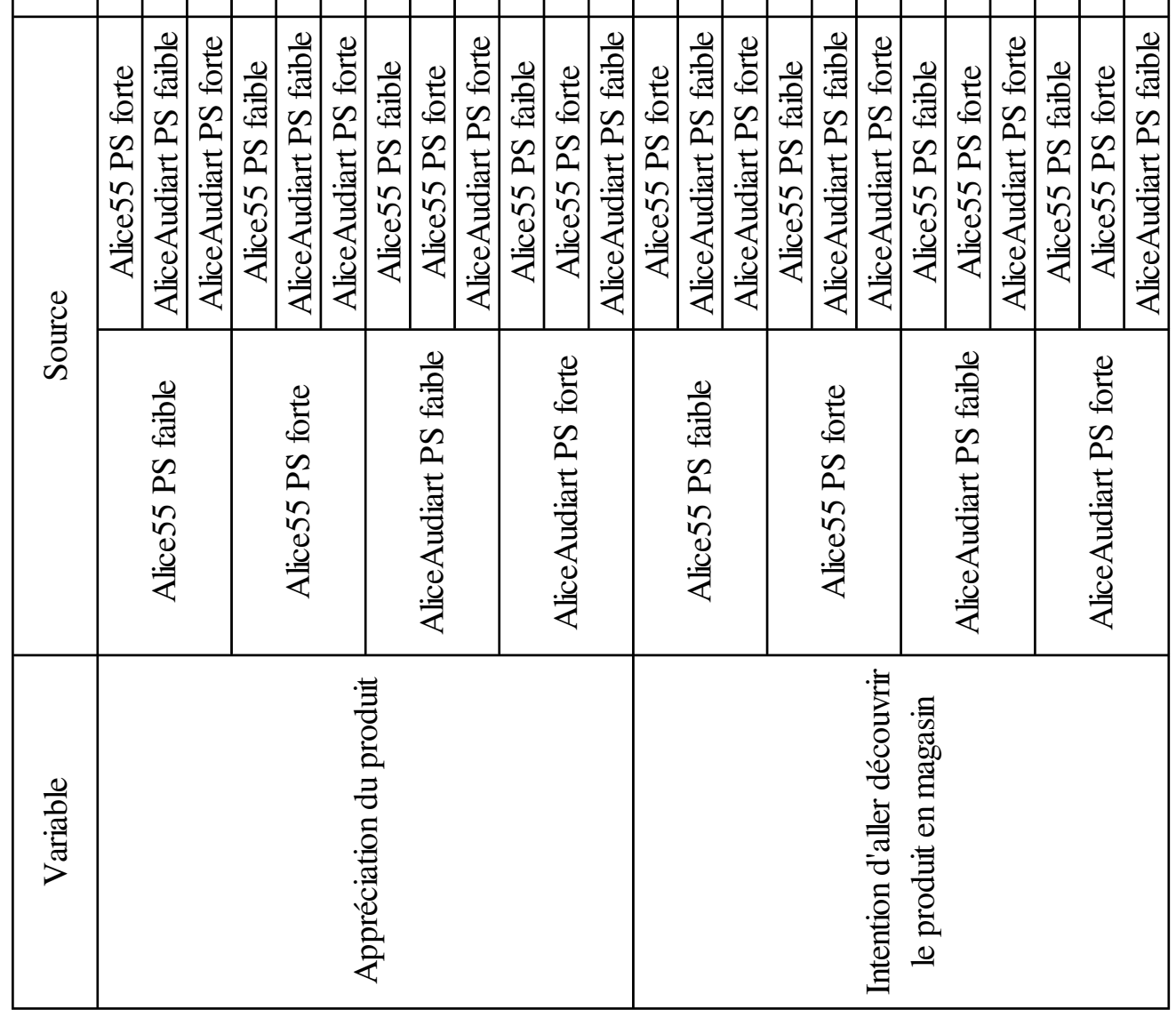




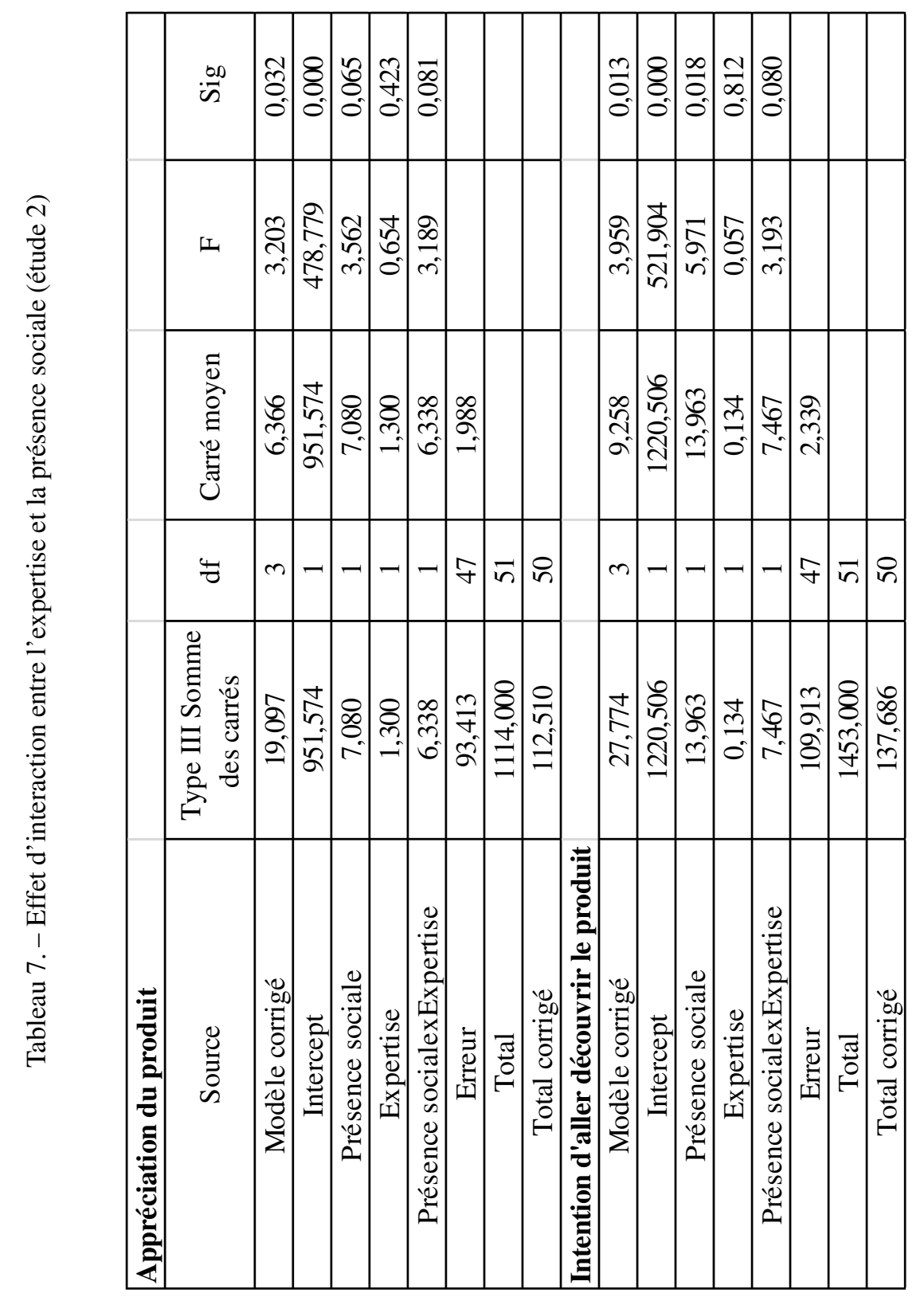




\section{Discussion des résultats de la $2^{e}$ étude}

Comme dans la première étude, le pouvoir d'influence de la recommandation ne varie pas significativement selon qu'elle émane d'une source institutionnelle ou d'un internaute. Le levier de la persuasion réside plus dans l'impression de présence sociale qui se dégage de la recommandation que dans le degré d'expertise de la source. Les recommandations d'internautes ne peuvent remplacer les contenus institutionnels que pour les internautes lecteurs qui se sentent plus proches psychologiquement des internautes contributeurs que des sources officielles. Dans ce cas, les informations données par les internautes sont plus pertinentes et plus crédibles que celles données par les sources officielles. Pour les internautes qui se sentent plus proches des sources institutionnelles, les contenus institutionnels sont aussi influents que les recommandations d'internautes.

\section{CONCLUSIONS, LIMITES \\ ET VOIES DE RECHERCHE}

Notre travail de recherche visait à mettre en perspective le pouvoir d'influence des contenus générés par les utilisateurs et celui des contenus institutionnels sur les attitudes des lecteurs de blogs. La capacité des recommandations d'internautes à déclencher des attitudes favorables à l'égard des produits présentés sur les blogs était comparée à celle des sources institutionnelles (incarnées ou désincarnées). L'intérêt de l'article était d'appréhender l'impression de présence sociale que les internautes percevaient dans les recommandations en ligne. Cette approche individuelle, nouvelle dans le champ des Media Research, est porteuse de plusieurs résultats intéressants.

Cette recherche montre que, sur un blog, le crédit accordé à une recommandation dépend de la relation qui se crée entre l'internaute contributeur et l'internaute lecteur. Un lecteur se laisse d'autant plus convaincre par une recommandation qu'il ressent de la présence sociale dans la recommandation, c'est-à- dire, au regard de la définition originelle de Short, Williams et Christie (1976), une forme de proximité psychologique (sociale, spatiale, temporelle) avec l'internaute contributeur. Ainsi, l'internaute lecteur doit avoir l'impression qu'il discute, à travers le blog, avec une personne qui lui ressemble, qui partage les mêmes opinions que lui, et qui lui donne des conseils pertinents au regard de ses attentes. Si tel est le cas, alors la recommandation suscite une attitude plus favorable à l'égard du produit qu'un contenu institutionnel désincarné, comme un article de type journalistique, qui est faible en présence sociale. Dans le cas contraire, elle déclenche une attitude à l'égard du produit qui est équivalente à celle engendrée par l'article de type journalistique. Ainsi, la supériorité des recommandations d'internautes par rapport aux contenus institutionnels n'est avérée que pour les internautes lecteurs qui se sentent plus proches psychologiquement de l'internaute contributeur que de la source officielle (marque, journaliste ou spécialiste). Tous les autres internautes accorderont un crédit relativement équivalent aux informations fournies par les internautes et celles fournies par les sources institutionnelles. Ainsi il faut donc nuancer la croyance que, sur les médias sociaux, les contenus générés par les utilisateurs, supposés non experts, prennent le pas sur les sources institutionnelles, supposées expertes. La proximité avec la source est un plus fort levier de persuasion que l'expertise.

Nos résultats comportent également certaines limites qui invitent, à l'avenir, à compléter cette recherche. Tout d'abord, il est intéressant de noter que l'effet de la présence sociale est non significatif pour la recommandation d'Alice55 mais significatif pour les recommandations de Marie88 et d'Alice Audiart. Alice55 et Marie88 étant aussi peu expertes du produit l'une que l'autre, ces résultats suggèrent l'existence d'une autre variable. La littérature indique que les contenus générés par les internautes sont d'autant plus privilégiés que le consommateur est novice (Chen et Xie, 2008). En effet, les consommateurs ont l'impression que le parfum ressemble plus aux parfums qu'ils ont l'habitude de porter lorsqu'il est recommandé par Alice55 que par Marie88 et Alice Audiart $(\mathrm{p}<0,05)$. Il sera intéressant de montrer dans une prochaine étude que la présence sociale ressentie dans une recommandation améliore d'autant moins l'attitude à l'égard du produit que le consommateur se sent expert du produit. 
Par ailleurs, l'analyse des tendances non significatives soulève quelques questions. Dans l'étude 1, la recommandation de Marie88, lorsqu'elle dégage une faible présence sociale, entraîne une moins bonne attitude à l'égard du produit que l'article de type journalistique. Le contenu institutionnel à faible présence sociale est alors plus persuasif que le contenu généré par les internautes à faible présence sociale. À l'inverse, dans l'étude 2, la recommandation d'Alice55, lorsqu'elle dégage une faible présence sociale, engendre une meilleure attitude à l'égard du produit que la recommandation d'Alice Audiart lorsqu'elle dégage une faible présence sociale. Le contenu institutionnel à faible présence sociale est alors moins persuasif que le contenu généré par les internautes à faible présence sociale. Même si ces différences sont trop faibles pour être significatives, il semble que les effets enregistrés puissent varier selon que le contenu institutionnel émane d'une personne humaine (un spécialiste, un expert) ou d'une entité désincarnée (une marque par exemple). Lorsque le produit est recommandé par une personne humaine, l'impression de se sentir éloigné (temporellement, spatialement ou socialement) pourrait déclencher un véritable rejet pour le produit. Cette question pourra faire l'objet d'une recherche ultérieure.

Plusieurs voies de recherche futures peuvent également être proposées. Premièrement, il serait intéressant d'étudier des contenus institutionnels sur d'autres médias sociaux, comme par exemple l'avis du leader d'opinion d'une communauté virtuelle, des tweets sur un microblogs ou encore des messages diffusés au sein de mondes virtuels dont la présence sociale est élevée (Kaplan et Haenlein, 2010). De plus, notre approche consistait, comme il est d'usage dans les recherches sur Internet, à opposer les commentaires incarnés, censés être des vecteurs de forte présence sociale, aux contenus désincarnés, censées être des sources d'informations institutionnelles dénuées de présence sociale. Toutefois, si les contenus désincarnés comportent généralement peu d'indices de présence sociale (voir une liste de ces indices en Annexe A2), certains internautes peuvent, notamment lorsqu'ils connaissent particulièrement bien le blog et qu'ils y sont attachés, ressentir une forme de contact humain dans les contenus désincarnés. Il serait ainsi intéressant de reconduire cette recherche sur un blog véhiculant des valeurs et une personnalité de marque. Il est également important de répliquer l'étude sur d'autres terrains d'application, et auprès d'un échantillon composé d'hommes et de femmes. Des produits moins impliquants, moins complexes à verbaliser, et dont les bénéfices sont d'ordre plus fonctionnels pourront être étudiés. En effet, on peut se demander dans quelle mesure l'impression de présence sociale varie selon la nature des bénéfices que le produit apporte : fonctionnels, hédoniques ou symboliques (Park et alii, 1986). En dernier lieu, bien qu'elles soient rarement étudiées dans ce champ de recherche, exception faite pour le genre et le degré d'acceptation de la technologie, les variables influençant la sensibilité des internautes aux différents vecteurs de présence sociale (Annexe A2) ou aux indices censés renforcer la crédibilité du membre contributeur (e.g. le statut contributeur ${ }^{16}$ ) devront être explorées à l'avenir. Certaines caractéristiques individuelles des internautes lecteurs, comme par exemple le niveau de self-monitoring ou l'extraversion, pourraient renforcer la tendance des internautes à accorder du crédit aux contributions sur les blogs. Une meilleure connaissance de ces déterminants aidera notamment les praticiens à définir leur politique de modération des contributions, à choisir les indices les plus pertinents pour renforcer l'impression de présence sociale ou l'expertise du membre contributeur, à adopter la bonne répartition entre les contributions de membres et les contenus institutionnels.

\section{RÉFÉRENCES BIBLIOGRAPHIQUES}

Aberg J. et Shahmehri N. (2001), An empirical study of human web assistants: implications for user support in web information systems, SIGCHI'O1, Seattle.

Allen P.A., McNeal M. et Kvak D. (1992), Perhaps the lexicon is coded as a function of word frequency, Journal of Memory and Language, 31, 6, 826-844.

Ansari S., Essegaier S. et Kohli R. (2000), Internet recommendations systems, Journal of Marketing Research, $37,3,363-375$.

16. Système par lequel le contributeur d'un forum se voit attribuer successivement différents statuts honorifiques en fonction du nombre de contributions réalisées (contributeur Gold, Silver sur Ciao.fr ou acheteur certifié sur Laredoute.fr.). 
Balagué C. et Florès L. (2007), Buzz on line : quel impact sur les entrées des films de cinéma?, $23^{e}$ Congrès international de l'Association Française du Marketing, Aix-les-Bains.

Belvaux B. et Marteaux S. (2007), Les recommandations d'internautes comme source d'information: Quel impact sur les entrées des films au cinéma ?, Recherche et Applications en Marketing, 22, 3, 65-82.

Bickart B. et Schindler R.M. (2001), Internet forums as influential sources of consumer information, Journal of Interactive Marketing, 15, 3, 31-40.

Biocca F., Burgoon J.K., Harms C. et Stonner M. (2001), Criteria and scope conditions for a theory and measure of social presence, Actes de la Conférence Présence 2001, Philadelphie, www.mindlab.org

Biocca F., Harms C. et Burgoon J.K. (2003), Criteria for a theory and measure of social presence, Presence: Teleoperators and Virtual Environments, 12, 5, 456-480.

Bounie D., Bourreau M., Gensollen M. et Waelbroeck P. (2008), Do online customer reviews matter? Evidence from the video game industry, Working Papers in Economics and Social Sciences, ESS-08-02, Telecom ParisTech.

Bronner F. et de Hoog R. (2010), Consumer-generated versus marketer-generated websites in consumer decision making, International Journal of Market Research, 52, 2, 231-248.

Brown J.J. et Reingen P.H. (1987), Social ties and word-ofmouth referral behavior, Journal of Consumer Research, 14, 3, 350-362.

Buhalis D. et Law R. (2008), Progress in information technology and tourism management: 20 years on and 10 years after the internet - the state of eTourism research, Tourism Management, 29, 609-623

Burke K. et Chindambaram L. (1999), How much bandwidth is enough? A longitudinal examination of media characteristics and group outcomes, MIS Quarterly, 23, $4,557-580$.

Cassaigne B. (2010), Le e-commerce a tout changé !, Paris, 6e Baromètre FEVAD - Médiamétrie // Net Ratings, A.G. FEVAD.

Chen Y. et Xie J. (2008), Online consumer review: wordof-mouth as a new element of marketing communication mix, Management Science, 54, 3, 477-491.

Chevalier J.A. et Mayzlin D. (2006), The effect of word of mouth on sales: online book reviews, Journal of Marketing Research, 43, 3, 345-354.

Clemons E. K., Gao G. et Hitt L.M. (2006), When online reviews meet hyperdifferentiation: a study of the craft beer industry, Journal of Management Information Systems, 23, 2, 149-171.

Cyr D., Hassanein K., Head M. et Ivanov A. (2007), The role of social presence in establishing loyalty in e-service environments, Interacting with computers, 19, 1, 43-56.

Dash S. et Saji K.B. (2007), The role of consumer self-efficacy and website social presence in customers' adoption of B2C online shopping: an empirical study in the Indian context, Journal of International Consumer Marketing, 20, 2, 33-48.
Davis F.D. (1989), Perceived usefulness. Perceived ease of use and user acceptance of information technology, MIS Quarterly, 13, 3, 319-340.

De Greef P. et Ijsselsteijn W. (2000), Social presence in the photoshare teleapplication, Third International Presence Workshop, Techniek Museum, Delft.

Desrochers A., Paivio A. et Desrochers S. (1989), L'effet de la fréquence d'usage des mots inanimés et de la valeur prédictive de leur terminaison sur l'identification du genre grammatical, Revue Canadienne de Psychologie, 43, 1, 62-73.

Duan W., Gu B. et Whinston A.B. (2008), Do online reviews matter? An empirical investigation of panel data, Decision Support Systems, 45, 4, 1007-1016.

Eccleston D. et Griseri L., How does Web 2.0 stretch traditional influencing patterns?, International Journal of Market Research, 50, 5, 591-616.

Ellis A.W. et Miller D. (1981), Left and wrong in adverts: neuropsychological correlates of aesthetic preference, British Journal of Psychology, 72, 2, 225-230.

Featherman M.S., Valacich J.S. et Wells J.D. (2006), Is that authentic or artificial? Understanding consumer perceptions of risk in e-service encounters, Information Systems Journal, 16, 2, 107-134.

Feick L. et Higie R.A. (1992), The effect of preference heterogeneity and source characteristics on ad processing and judgments about endorsers, Journal of Advertising, $21,2,9-24$.

Firat A.F., Dholakia N. et Venkatesh A. (1995), Marketing in a post modern world, European Journal of Marketing, $29,1,40-56$.

Frambach R.T., Roest H.C.A. et Krishnan T.V. (2007), The impact of consumer internet experience on channel preference and usage intentions across different stages of the buying process, Journal of Interactive Marketing, 21, 2, 26-41.

Fuchs C., Prandelli E. et Schreier M. (2010), The psychological effects of empowerment strategies on consumers'product demand, Journal of Marketing, 74, 1, 65-79.

Gefen D. et Straub D.W. (1997), Gender differences in the perception and use of email: an extension of the TAM, MIS Quarterly, 21, 4, 389-400.

Gefen D. et Straub D.W. (2003), Trust and TAM in online shopping: an integrated model, MIS Quarterly, 27, 1, 51-90.

Gefen D. et Straub D.W. (2004), Consumer trust in B2C eCommerce and the importance of social presence: experiments in e-Products and e-Services, The International Journal of Management Science, 32, 6, 407-424.

Hass R.G. (1981), Effects of source characteristics on cognitive response and persuasion, in R.E. Petty, T.M. Ostrom et T.C. Brock (coord.), Cognitive responses in persuasion, Hillsdale, NJ, Lawrence Erlbaum Associates.

Hassanein K. et Head M. (2005), The impact of infusing social presence in the Web interface: an investigation across product types, International Journal of Electronic Commerce, 10, 2, 31-55. 
Hassanein K. et Head M. (2007), Manipulating perceived social presence through the web interface and its impact on attitude towards online shopping, International Journal of Human-Computer Studies, 65, 5, 689-708.

Helme-Guizon A. (2001), Le comportement du consommateur sur un site marchand est-il fondamentalement différent de son comportement en magasin ? Proposition d'un cadre d'appréhension de ses spécificités, Recherche et Applications en Marketing, 16, 3, 25-38.

Janiszewski C. (1988), Preconscious processing effects: the independence of attitude formation and conscious thought, Journal of Consumer Research, 15, 2, 199-209.

Kaplan A.M. et Haenlein M. (2010), Users of the world, unite! The challenges and opportunities of social media, Business Horizons, 53, 1, 59-68.

Keller P.A. et Block L.G. (1997), Vividness effects: a resource matching perspective, Journal of Consumer Research, 24, 3, 295-304.

Komiak S.Y.X. et Benbasat I. (2006), The effects of personalization and familiarity on trust and adoption of recommendation agents, MIS Quarterly, 30, 4, 941960.

Kumar N. et Benbasat I. (2006), The influence of recommendations and consumer reviews on evaluations of websites, Information Systems Research, 17, 4, 425439.

Kumar N. et Benbasat I. (2002a), Para-social presence and communication capabilities of a web site, e-Service Journal, 1, 3, 5-24.

Kumar N. et Benbasat I. (2002b), Para-Social Presence: a reconceptualization of «Social Presence » to capture relationship between a web site and her visitors, Proceedings of the $35^{\text {th }}$ Hawaii International Conference on System Sciences.

Larceneux F. (2007), Buzz et recommandations sur Internet : quels effets sur le box-office ?, Recherche et Applications en Marketing, 22, 3, 46-64.

Lombard M. et Ditton T. (1997), At the heart of it all: the concept of presence, Journal of Computer-Mediated Communication (on line), 3, 2 , http://jcmc.indiana.edu/vol3/issue2/lombard.html.

McGuire W.J. (1969), The nature of attitudes and attitude change, in G. Lindzey et E. Aronson (coord.), The handbook of social psychology (2e édition), 3, 136-314.

Monsell S., Doyle M.C. et Haggard P.N. (1989), Frequency effects in visual word recognition: where are they?, Journal of Experimental Psychology: General, 118, 1, 43-71.

Nass C. et Steuer J. (1993), Voices, boxes, and sources of messages: computers and social actors, Human Communication Research, 19, 4, 504-527.

Paap K.R. et Johansen L.S. (1994), The case of the vanishing frequency effect: a retest of the verification model, Journal of Experimental Psychology : Human Perception and Performance, 20, 6, 1129-1157.

Palmer M.T. (1995), Interpersonal communication and virtual reality: mediating interpersonal relationships, in
F. Biocca et M.R. Levy (coord.), Communication in the age of virtual reality, Hillsdale, NJ, Lawrence Erlbaum Associates, Publishers.

Papadopoulou P., Andreou A., Kanellis P. et Martakos D. (2001), Trust and relationship building in electronic commerce, Internet Research: Electronic Networking Applications and Policy, 11, 4, 322-332.

Park C.W., Jaworski B.J. et McInnis D.J. (1986), Strategic brand concept-image management, Journal of Marketing, 50, 3, 135-145.

Pavlou P., Liang H. et Xue Y. (2005), Understanding and mitigating uncertainty in online environments: a longitudinal analysis of the role of trust and social presence, Best Paper Proceedings of the Academy of Management Conference, OCIS Division Best Paper Award.

Pisani F. et Piotet D. (2008), Comment le Web change le monde, l'alchimie des multitudes, Paris, Pearson Education France.

Prahalad C.K. et Ramaswamy V. (2004), Co-creation experiences: the next practice in value creation, Journal of Interactive Marketing, 18, 3, 5-14.

Price L.L., Feick L.F. et Higie R.A. (1989), Preference heterogeneity and coorientation as determinants of perceived informational influence, Journal of Business Research, 19, 3, 227-242.

Rebillard F. (2007), Le web 2.0 en perspective, Paris, L'Harmattan.

Reeves B. et Nass C. (1996) [2002], The media equation: how people treat computers, television and new media like real people and places, Stanford, CA, CSLI Publications.

Rice R.E. (1992). Task analyzability, use of new medium and effectiveness: a multi-site exploration of media richness, Organization of Science, 3, 4, 475-500.

Sallnas E., Rassmus-Grohn K. et Sjöström C. (2000), Supporting presence in collaborative environments by haptic force feedback, AMC Transactions on Human Computer Interaction, 7, 461-476.

Senecal S. et Nantel J. (2004), The influence of online product recommendations on consumers' online choices, Journal of Retailing, 80, 2, 159-169.

Short J., Williams E. et Christie B. (1976), The social psychology of telecommunications, Londres, John Wiley and Sons.

Smith D., Menon S. et Sivakumar K. (2005), Online peer and editorial recommendations, trust and choice in virtual markets, Journal of Interactive Marketing, 19, 3, 15-37.

Steinfield C. (1986). Computer-mediated communication in an organizational setting: explaining task-related and socioemotional cues, in M. McLaughlin (coord.), Communication yearbook, Newbury Park, CA, Sage Publications.

Surowiecki J. (2005), La sagesse des foules, Paris, Lattès.

Tapscott D. et Williams D. (2007), Wikinomics, comment l'intelligence collaborative bouleverse l'économie, Village Mondial, Paris, Pearson Education France.

Tractinsky N. et Rao V.S. (2001), Incorporating social dimensions in web-store design, Human Systems Management, 20, 2, 105-121. 
Van Dolen W.M., Dabholkar P.A. et de Ruyter K. (2007), La satisfaction envers les discussions en ligne de clients : l'influence des attributs technologiques perçus, des caractéristiques du groupe de discussion et du style de communication du conseiller, Recherche et Applications en Marketing, 22, 3, 83-111.

Wang L.C., Baker J., Wagner J.A. et Wakefield K. (2007), Can a retail web site be social?, Journal of Marketing, 71, 3, 143-157.

Wiener M. et Mehrabian A. (1968), Language within language: immediacy, a channel in verbal communication, New York, Appleton Century Crofts.
Wilson E.J. et Sherrell D.L. (1993), Sources effects in communication and persuasion research: a meta-analysis of effect size, Journal of the Academy of Marketing Science, 21, 2, 101-112.

Woodside A.G. et Davenport J.W. Jr. (1974), The effect of salesman similarity and expertise on consumer purchasing behavior, Journal of Marketing Research, 11, 2, 198-202. 


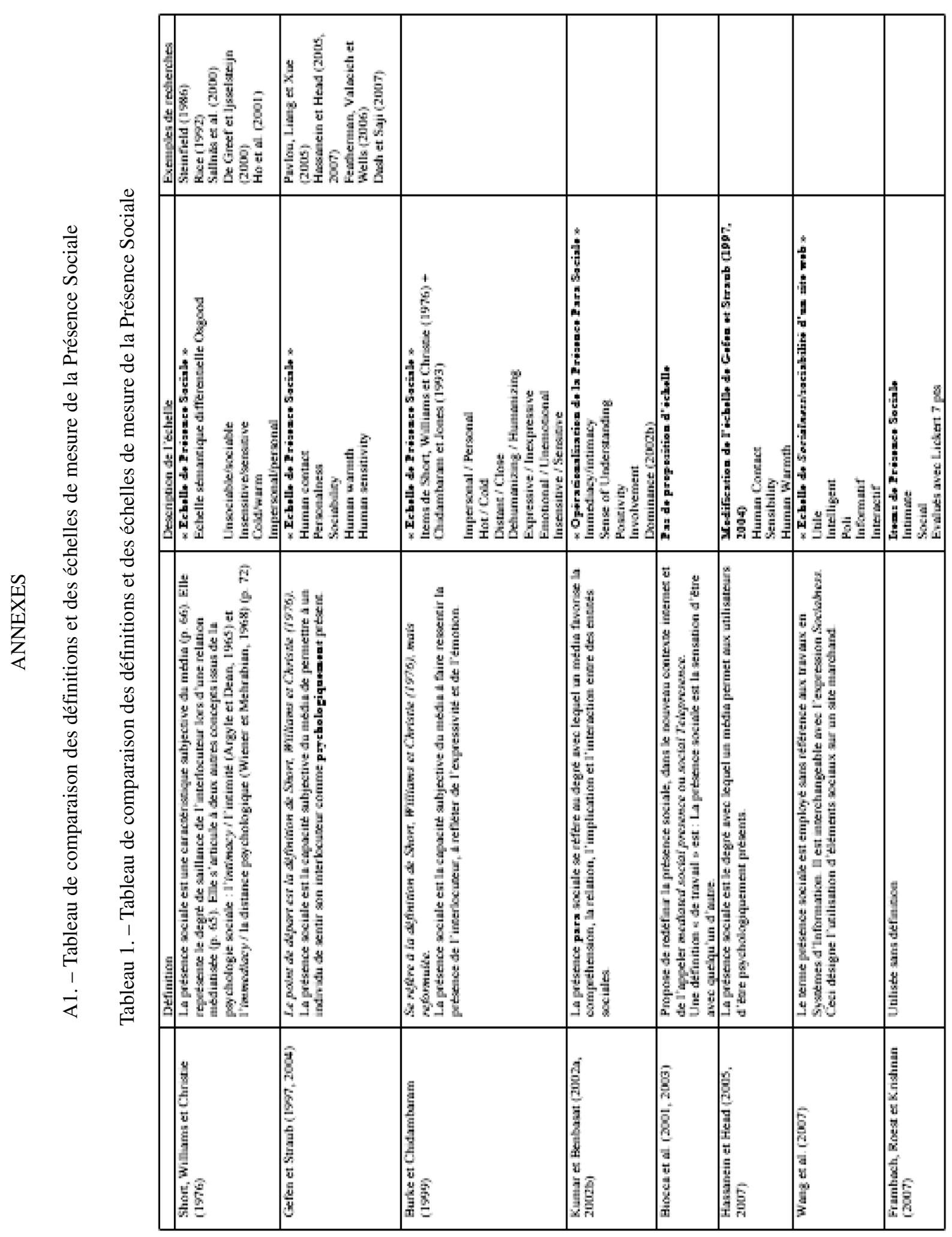


A2. - Les vecteurs de présence sociale sur les sites Internet

\begin{tabular}{|c|c|c|}
\hline \multicolumn{2}{|c|}{ Type de vecteurs de présence sociale } & Détail du vecteur de présence sociale \\
\hline \multicolumn{2}{|c|}{ Vecteurs anthropomorphiques } & $\begin{array}{l}\text { Agent virtuel (Aberg et Shahmehri, } 2001 \text {; } \\
\text { Papadopoulou et alii, 2001) } \\
\text { Photo, vidéo (Gefen et Straub, } 2003 \text {; } \\
\text { Kumar et Benbasat, 2002a) } \\
\text { Voix humaines (Lombard et Ditton, 1997) }\end{array}$ \\
\hline \multirow[t]{3}{*}{$\begin{array}{l}\text { Vecteurs non } \\
\text { anthropomorphiques }\end{array}$} & Vecteurs éditoriaux & $\begin{array}{l}\text { Texte : registre de langage au travers des } \\
\text { choix lexicaux et syntaxiques (Gefen et } \\
\text { Straub, } 2003 \text {; Nass et Steuer, 1993) } \\
\text { Mise en forme: polices, encadrés, } \\
\text { animations de type pop up, couleurs }\end{array}$ \\
\hline & $\begin{array}{l}\text { Vecteurs « marketing } \\
\text { direct » }\end{array}$ & $\begin{array}{l}\text { Envoi d'e-mails de la commande à la } \\
\text { livraison (Gefen et Straub, } 2003 \text {; Kumar } \\
\text { et Benbasat, 2002a) } \\
\text { Salutation du client par son nom, prénom } \\
\text { (Gefen et Straub, 2004; Kumar et } \\
\text { Benbasat, 2002a) }\end{array}$ \\
\hline & Vecteurs « Web» & $\begin{array}{l}\text { Avis de consommateurs (Kumar et } \\
\text { Benbasat, 2006), forums (Cyr et alii, } \\
\text { 2007), chats (Kumar et Benbasat, 2002a), } \\
\text { Moteurs de recommandation de produits } \\
\text { Web call center (chat avec un vendeur) }\end{array}$ \\
\hline
\end{tabular}


A3. - Textes descriptifs du parfum pour chaque condition expérimentale

\begin{tabular}{|c|c|c|}
\hline Étude 1 & Article de type journalistique & $\begin{array}{c}\text { Avis d'internaute } \\
\text { Contribution de Marie88 }\end{array}$ \\
\hline $\begin{array}{l}\text { Fragrance à dominante } \\
\text { pomme verte }\end{array}$ & $\begin{array}{l}\text { «a fragrance fleurie, douce et } \\
\text { fruitée s'ouvre sur la fraîcheur d'un } \\
\text { accord 'sorbet de pomme verte'. } \\
\text { Le cœur fleuri tout en légèreté } \\
\text { révèle une note de pivoine et un } \\
\text { accord 'bouton de rose'. En fond, } \\
\text { les bois s'adoucissent de notes } \\
\text { ambrées et musquées.» }\end{array}$ & $\begin{array}{l}\text { «Au départ, c'est comme un sorbet } \\
\text { de pomme verte ! Puis on sent des } \\
\text { fleurs (pivoine... ou rose ?!?). } \\
\text { Lorsqu'on le porte plus longtemps, } \\
\text { il devient plus boisé (plus } \\
\text { ambré ?)... En fait, c'est à la fois } \\
\text { fleuri, et fruité ! C'est doux...» }\end{array}$ \\
\hline Étude 2 & $\begin{array}{c}\text { Avis d'internaute } \\
\text { Contribution d'Alice55 }\end{array}$ & $\begin{array}{c}\text { Avis de spécialiste } \\
\text { Contribution d'Alice Audiard }\end{array}$ \\
\hline $\begin{array}{l}\text { Fragrance à dominante de } \\
\text { cocktail de fruits }\end{array}$ & $\begin{array}{l}\text { La fragrance évoque un cocktail de } \\
\text { fruits frais, acidulé et sucré. Elle } \\
\text { dévoile tout de suite le citron, la } \\
\text { clémentine et l'orange puis, en } \\
\text { cœ ur, des notes de fruits rouges. } \\
\text { C'est un parfum coloré qui sent les } \\
\text { beaux jours. }\end{array}$ & $\begin{array}{l}\text { «Imaginez un cocktail de fruits } \\
\text { frais, acidulé et sucré... on } \\
\text { reconnaît tout de suite le citron, la } \\
\text { clémentine et l'orange. Ensuite, on } \\
\text { devine également des notes de } \\
\text { fruits rouges. C'est coloré... Ça } \\
\text { sent les beaux jours!» }\end{array}$ \\
\hline
\end{tabular}




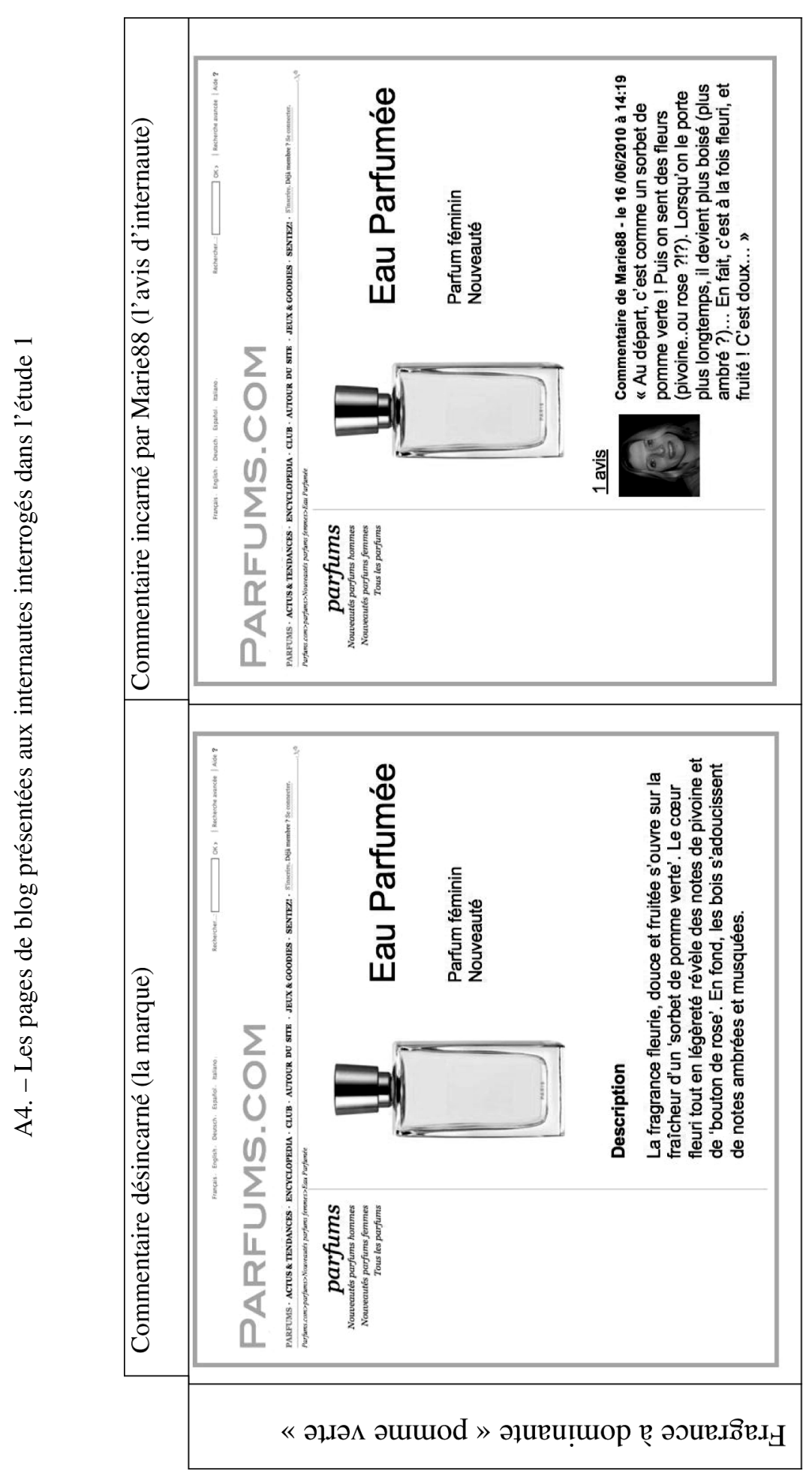




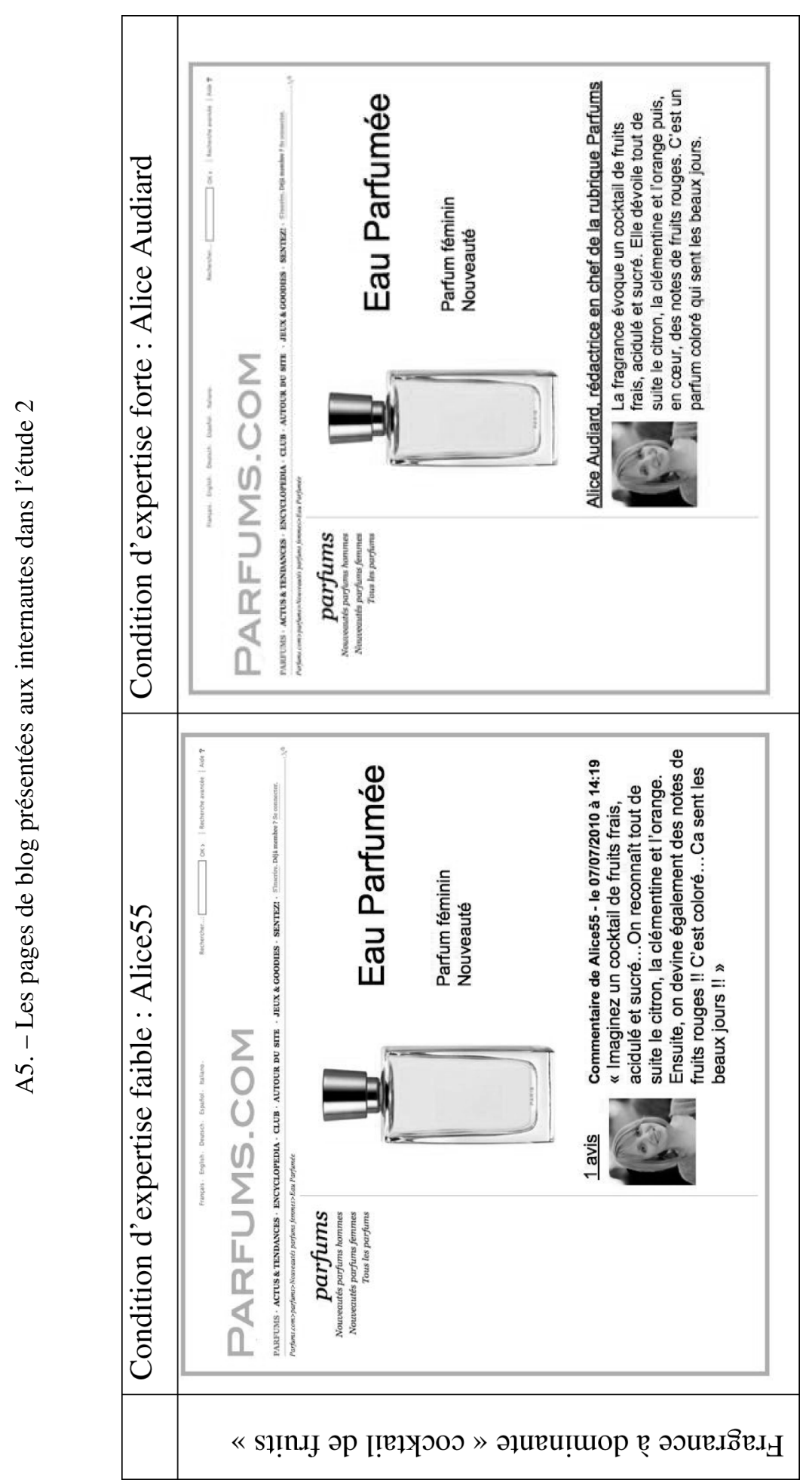


A6. - L'échelle de présence sociale (Gefen et Straub, 2004)

\begin{tabular}{|l|c|c|c|c|c|c|c|}
\hline & $\begin{array}{c}\text { Pas du tout } \\
\text { d'accord }\end{array}$ & & & & & & $\begin{array}{c}\text { Tout à fait } \\
\text { d'accord }\end{array}$ \\
\hline Il y a des contacts humains & & & & & & & \\
\hline On s'adresse à moi personnellement & & & & & & & \\
\hline Il y a de la convivialité & & & & & & & \\
\hline Il y a de la chaleur humaine & & & & & & & \\
\hline $\begin{array}{l}\text { Il se dégage une certaine sensibilité } \\
\text { humaine }\end{array}$ & & & & & & & \\
\hline
\end{tabular}


Ardelet-Brial 29/09/11 14:04 Page 26 